\title{
Faithful anaphase is ensured by Mis4, a sister chromatid cohesion molecule required in $S$ phase and not destroyed in $G_{1}$ phase
}

\author{
Kanji Furuya, Kohta Takahashi, and Mitsuhiro Yanagida ${ }^{1}$ \\ CREST Research Project, Department of Biophysics, Graduate School of Science, Kyoto University, \\ Kitashirakawa-oiwake-cho, Sakyo-ku, Kyoto 606, Japan
}

\begin{abstract}
The loss of sister chromatid cohesion triggers anaphase spindle movement. The budding yeast Mcd1/Scc1 protein, called cohesin, is required for associating chromatids, and proteins homologous to it exist in a variety of eukaryotes. Mcd1/Scc1 is removed from chromosomes in anaphase and degrades in $G_{1}$. We show that the fission yeast protein, Mis4, which is required for equal sister chromatid separation in anaphase is a different chromatid cohesion molecule that behaves independent of cohesin and is conserved from yeast to human. Its inactivation in $G_{1}$ results in cell lethality in $S$ phase and subsequent premature sister chromatid separation. Inactivation in $G_{2}$ leads to cell death in subsequent metaphase-anaphase progression but missegregation occurs only in the next round of mitosis. Mis4 is not essential for condensation, nor does it degrade in $\mathrm{G}_{1}$. Rather, it associates with chromosomes in a punctate fashion throughout the cell cycle. mis 4 mutants are hypersensitive to hydroxyurea $(\mathrm{HU})$ and $\mathrm{UV}$ irradiation but retain the ability to restrain cell cycle progression when damaged or sustaining a block to replication. The mis4 mutation results in synthetic lethality with a DNA ligase mutant. Mis4 may form a stable link between chromatids in S phase that is split rather than removed in anaphase.
\end{abstract}

[Key Words: Cell cycle; cohesin; fission yeast; mitosis; UV irradiation; hydroxyurea]

Received July 27, 1998; revised version accepted September 9, 1998.

In eukaryotic cells, replication of chromosomal DNA occurs once during $\mathrm{S}$ phase followed by separation of duplicated DNAs into two daughter nuclei during M-phase. DNA replication is initiated at the onset of $S$ phase in particular chromosomal regions, the origins, which are unwound and stabilized by single-strand-binding protein. The replication machinery is large and complex, containing a number of enzyme subunits including DNA polymerases and helicase. However, other factors are necessary for initiating replication such as the origin recognition complex, which is bound to origin sequences throughout the cell cycle. Cdc6 and the MCM complex are also needed and are loaded onto the origins to form the prereplicative complex (e.g., see Rowles and Blow 1997). Moreover, an essential protein kinase Cdc7/Dbf4 must act prior to replication, so that the replication machinery can interact with the prereplicative complex and thereby ensure continuing replication. The proteins described above are still part of the gene products required for the quality control of DNA replication. When chromosomal DNAs are damaged or replication is blocked,

${ }^{1}$ Corresponding author.

E-MAIL yanagida@kozo.biophys.kyoto-u.ac.jp; FAX 81757534208. entry into mitosis is restrained until the damage is recognized and repaired (e.g., see Kitazono and Matsumoto 1998). A block of mitotic entry upon damage or inhibition of replication can be accomplished by inactivation of cyclin-dependent protein kinases (CDKs). This checkpoint system is important for maintaining the genome with high fidelity, otherwise damaged chromosomal information will be transmitted to the nuclei of progeny cells.

To maintain the genome at high fidelity, there is an enigmatic aspect in chromosomal structure, that is, the mechinism by which sister chromatids are linked after duplication of chromosomal DNA. This sister chromatid link, alternatively called sister chromatid cohesion, must be dissociated before chromosomes are segregated into daughter cells (Holloway et al. 1993). Sister cohesion is thought to oppose the splitting forces exerted by kinetochore microtubules. If such linkage is absent, precocious sister chromatid separation will take place. The loss of sister cohesion in normal anaphase may thus trigger spindle movement. Until quite recently, very little or nothing has been known about the proteins required for sister chromatid cohesion. The first such protein to be identified was the Drosophila MEI-S332 protein required 
for the maintenance of sister chromatid cohesion in male and female meiosis (Kerrebrock et al. 1995). This protein localizes to meiotic and mitotic centromeres (although it is not essential for mitosis) until sister chromatids separate and are dissociated from chromosomes at anaphase (Moore et al. 1998), suggesting that the destruction or the release of this protein might be required for sister chromatid separation. In fission yeast, meiotic sister chromatid cohesion is deficient in the recombination-defective rec8-110 mutant (Molnar et al. 1995). Aberrant segregation is caused by precocious segregation of sister chromatids at anaphase I. In the same organism, mitotic centromeres are separated in metaphase-arrested mis6 mutants defective in equal segregation of sister chromatids in mitosis (Saitoh et al. 1997). Mis6 is essential for the formation of a centromere-specific chromatin in the $G_{1} / S$ phase and maintains the connection of sister centromeres until anaphase.

In budding yeast, Mcd1p/Scclp, a sister-chromatid cohesion protein called cohesin, was identified by genetic screening and found to form the complex with Smclp, an SMC protein, known to be involved in the structural maintenance of chromosomes (Guacci et al. 1997; Michaelis et al. 1997). Mcdlp/Scclp is similar to fission yeast Rad21 (Birkenbihl and Subramani 1992) and Rec8, and proteins homologous to Mcd1p/Scclp are present in higher eukaryotes. The mcd1 mutant is defective in sister chromatid cohesion and chromosome condensation. Scclp associates with chromosomes in an Smclp-dependent manner, dissociates from chromosomes at the metaphase-to-anaphase transition, and is degraded in $\mathrm{G}_{1}$ by the anaphase promoting complex (APC)/cyclosome. Thus $\operatorname{Rad} 21 / \operatorname{Mcd} 1 \mathrm{p} /$ Scclp-like proteins may form a common cohesion apparatus that forms a complex with two SMC proteins and is regulated by association with and removal from chromosomes in the cell cycle. The other protein, Scc2p, was discovered to be required for sister chromatid cohesion in budding yeast (Michaelis et al. 1997). Its behavior in the cell cycle and relationship to the cohesin complex has not been reported.

In this report we show that the Mis4 protein, initially identified by its requirement for stable maintenance of minichromosomes in fission yeast (Takahashi et al. 1994), is required for sister chromatid cohesion, and its function is quite distinct from cohesin. Fission yeast mis mutants are temperature-sensitive lethal at the restrictive temperature $\left(36^{\circ} \mathrm{C}\right)$ and can grow at the permissive temperature $\left(26^{\circ} \mathrm{C}\right)$, but show high loss rates of minichromosomes at $26^{\circ} \mathrm{C}$ or semi-permissive temperatures. Twelve mis genes have been identified. The mis $5^{+}$gene encodes one of the MCM subunits essential for replication (Takahashi et al. 1994; Adachi et al. 1997) whereas mis $6^{+}$codes for a centromere protein (Saitoh et al. 1997). The $\mathrm{mis}^{+}$and $\mathrm{mis} \mathrm{O}^{+}$genes encode the subunits of DNA polymerase $\delta$ (H. Tatebe, H. Kondoh, M. Yanagida, unpubl.). The phenotype of mis4 is similar to that of mis6, as not only minichromosomes but also regular chromosomes were missegregated at $36^{\circ} \mathrm{C}$ (Takahashi et al. 1994). In addition to the mis phenotype, the mis 4 mutant displays a low frequency of the cut phenotype (the occurrence of septation or cytokinesis in the absence of normal nuclear division; Hirano et al. 1986), and hypersensitivity to hydroxyurea (HU) and UV irradiation at permissive and semi-permissive temperatures (Takahashi et al. 1994). Mis4 thus appears to play an important role in maintaining the quality of the genome against cell cycle aberration, damage, and replication inhibition.

\section{Results \\ Missegregation in mis4-242 is preceded by a prior lethal event}

To determine which stage is defective during the cell cycle, cultures of mis4-242 were synchronized. Small, early $G_{2}$ cells were collected by use of an elutriator rotor from an exponentially grown culture at $26^{\circ} \mathrm{C}$, the permissive temperature. Then the culture was transferred to $36^{\circ} \mathrm{C}$, the restrictive temperature (0 min, Fig. $\left.1 \mathrm{~A}\right)$. Viability of the mutant cells remained high until $60 \mathrm{~min}$ but then decreased and reached $50 \%$ at about $100 \mathrm{~min}$ while the cells proceeded across the first mitosis at around 80-100 min. To distinguish the timing of metaphase and anaphase, the frequencies of cells showing short or elongated spindles were measured; cells containing short spindles $(<2.5 \mu \mathrm{m}$ in length), which corresponded to prophase through metaphase, reached a maximum at $80 \mathrm{~min}$, whereas those displaying elongated anaphase spindles $(>2.5 \mu \mathrm{m}$ and up to $14 \mu \mathrm{m})$ peaked at 100 min. Thus mis4-242 cells synchronized culture in early $\mathrm{G}_{2}$ at $36^{\circ} \mathrm{C}$ appeared to lose viability as they progress from metaphase through anaphase. Further decrease of viability occurred in the succeeding $S$ phase and cell separation (around 120-140 $\mathrm{min}$ ). The $\mathrm{G}_{1}$ phase is very short in fission yeast.

In sharp contrast to the above observations, chromosome segregation and spindle movements were apparently normal during the first mitosis (micrographs in Fig. $1 \mathrm{~B}$ taken at $100 \mathrm{~min}$ : left, DNA stain; right, anti-tubulin antibody stain). Missegregation defects were quite frequently produced only later during the second round of mitosis at 200-220 min (Fig. 1C). Different types of missegregation are shown in Figure 1D (taken at $220 \mathrm{~min}$ ). Unequal segregation of sister chromatids was most common. Chromatids remaining along the anaphase spindle (indicated by arrowheads) were also seen. The kinetochore microtubules might fail to pull the chromatids toward the poles.

\section{mis4 mutants lose viability during $S$ phase}

We examined whether the missegregation defect could be produced in the first mitosis, if mutant cells previously arrested in $\mathrm{G}_{1}$ by nutrient starvation were transferred to the growing medium at $36^{\circ} \mathrm{C}$. Viability of the mutant cells sharply decreased between 3 and $4 \mathrm{hr}$ (Fig. 2A), while the cells traversed S phase (Fig. 2C, right panel). Abnormal missegregation phenotypes were frequently produced during the following first mitosis $(7 \mathrm{hr}$, 
Figure 1. A lethal event in the first cell cycle precedes the abnormal second mitosis in a $\mathrm{G}_{2}$-synchronized culture of mis4-242 cells. Mutant cells were grown at $26^{\circ} \mathrm{C}$, and small early $\mathrm{G}_{2}$ cells were collected by an elutriator rotor and then cultured at $36^{\circ} \mathrm{C}$ for $5 \mathrm{hr}$ in synthetic EMM2 medium. (A) Cell viability $(\% ; \boldsymbol{\Delta})$ was measured by plating of cells at $26^{\circ} \mathrm{C}$. Spindle indexes $(\%)$ were obtained by estimation of the frequencies of cells that contained the spindle stained by anti-tubulin antibody. The length of the spindle was classified into short $(<2.5 \mu \mathrm{m} ; \square)$ or long $(>2.5 \mu \mathrm{m} ; \mathbf{\square})$. Short spindles peaked at 80 and $200 \mathrm{~min}$, whereas long spindles peaked at 100 and $220 \mathrm{~min}$. The decrease of cell viability occurred when the two spindle indexes were high in the first mitosis. Abnormal mitosis occurred only in the second mitosis. (B) Mitotic cells in the first mitosis at $36^{\circ} \mathrm{C}$ showing normal anaphase. Micro

A

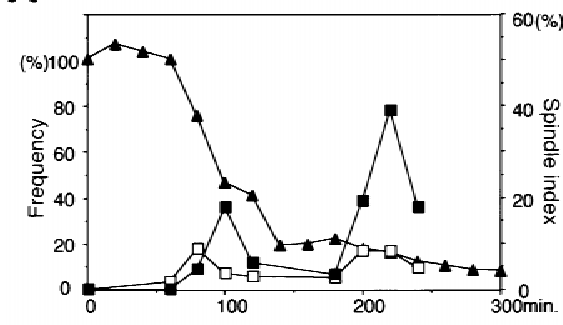

C

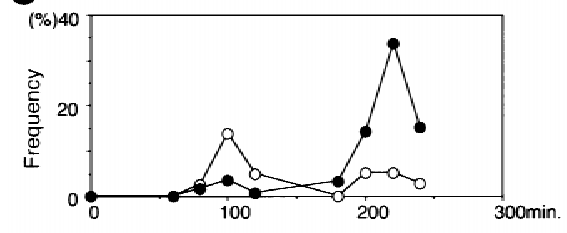

B

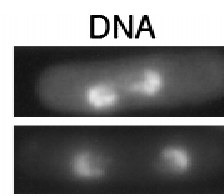

D

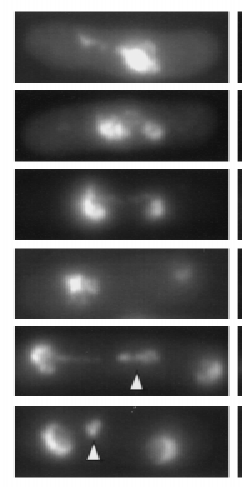

TUB
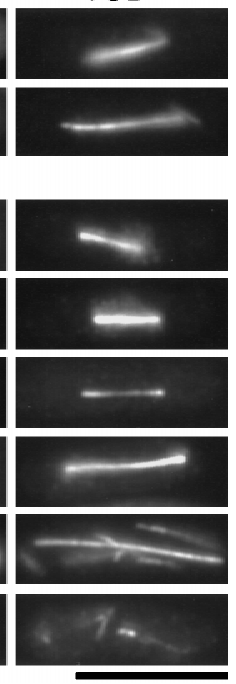

graphs taken at 100 min. (Left) Chromatin DNA staining by DAPI; (right) spindle staining by anti-tubulin antibody. (C) Frequencies of cells showing equal sister chromatid separation in normal anaphase $(O$; examples shown in $B)$ and unequal abnormal anaphase $(\boldsymbol{\bullet}$; examples in $D)$. (D) Mitotic cells in the second mitosis at $36^{\circ} \mathrm{C}$ showing unequal sister chromatid separation. Micrographs were taken at $220 \mathrm{~min}$. (Arrowheads) Chromatids remaining along the spindle. Bar, $10 \mu \mathrm{m}$.

Fig. 2B,D). These results showed that mis4-242 cells cultured from $\mathrm{G}_{1}$ at $36^{\circ} \mathrm{C}$ became inviable during the $\mathrm{S}$ phase, and chromosome missegregation occurred in the succeeding mitosis.

\section{The $160-\mathrm{kD}$ product of mis4 $4^{+}$is an evolutionarily conserved protein}

The mis $4^{+}$gene was isolated by a search of plasmids that fully suppressed the temperature-sensitive phenotype of mis4-242 by use of a genomic library of Schizosaccharomyces pombe. Thirty-nine independent clones were obtained, and they were all found to overlap, indicating that they were derived from a single genetic locus. The cloned DNA was integrated into the chromosome by homologous recombination with the $\mathrm{ura}^{+}$gene marker (see Materials and Methods), followed by tetrad analysis with the mis4-242 mutant, and verified to be derived from the mis $4^{+}$locus. The plasmid pKT221 was further subcloned, and the resulting pKF201 contained a minimal functional sequence $(5.1 \mathrm{~kb})$. The nucleotide sequence was determined, and one coding region representing the mis $4^{+}$gene was present. The predicted polypeptide contained 1583 amino acids interrupted with seven presumed introns (Fig. 3A). This coding region was also present in the $S$. pombe genome database.

A database search revealed that the fungus Coprinus cinereus Rad9 (2157 amino acids; Seitz et al. 1996) and the budding yeast Scc2 (1493 amino acids; Michaelis et al. 1997) showed a significant similarity from the central to the carboxy-terminal region of the polypeptides as illustrated in Figure 3B. Their overall identity was not high, but three regions showed significant sequence identity $(22 \%-38 \%)$. It was also noted that mouse and human cDNA sequences (HUMHBC4244 and AA062272) similar to the carboxy-terminal regions of these proteins exist in the databases, indicating that Mis4-like proteins are present in mammalian organisms.

The hemagglutinin antigen (HA) sequence was fused to the carboxyl terminus of Mis4. The resulting fusion protein was expressed in $S$. pombe cells either after integration into the chromosome or introduced as a multicopy plasmid. As shown in Figure 3C, HA-tagged Mis4 was detected at the expected molecular mass of $160 \mathrm{kD}$ (lane 2, integrated single copy; lane 3, overproducing plasmid). Nonintegrated cells carrying the vector plasmid did not show the band (lane 1). Comparison of the immunoblotting intensity of Cut2 also tagged with the same HA construct indicated that the amount of Mis4 in cells was approximately four-fold higher than Cut2, that is, $\sim 30,000$ molecules per cell.

In block and release experiments carried out with a cdc25 mutant, cells were arrested in late $\mathrm{G}_{2}$ and then released to the permissive temperature. The amount of Mis4 protein did not greatly fluctuate during the cell cycle; it showed an increase (1.5-fold) in late mitosis, but otherwise the level remained roughly constant. The control mitotic cyclin (Cdc13) decreased in exiting of mitosis (Fig. 3D). Mis4 did not degrade in the $G_{1}$-arrested cdc10 mutant cells, and the level did not change in the cyclosome subunit mutants (data not shown). Mis4 is thus present throughout the cell cycle, whereas budding yeast $\operatorname{Mcd} 1 \mathrm{p} / \mathrm{Sccl} \mathrm{p}$ is destroyed in $\mathrm{G}_{1}$.

\section{The mis4 $4^{+}$gene is essential for viability}

Gene disruption of mis $4^{+}$was achieved by a single-step replacement (see Materials and Methods). The coding region was replaced with the $S$. pombe $\mathrm{ura}^{+}$gene. The 
A

B
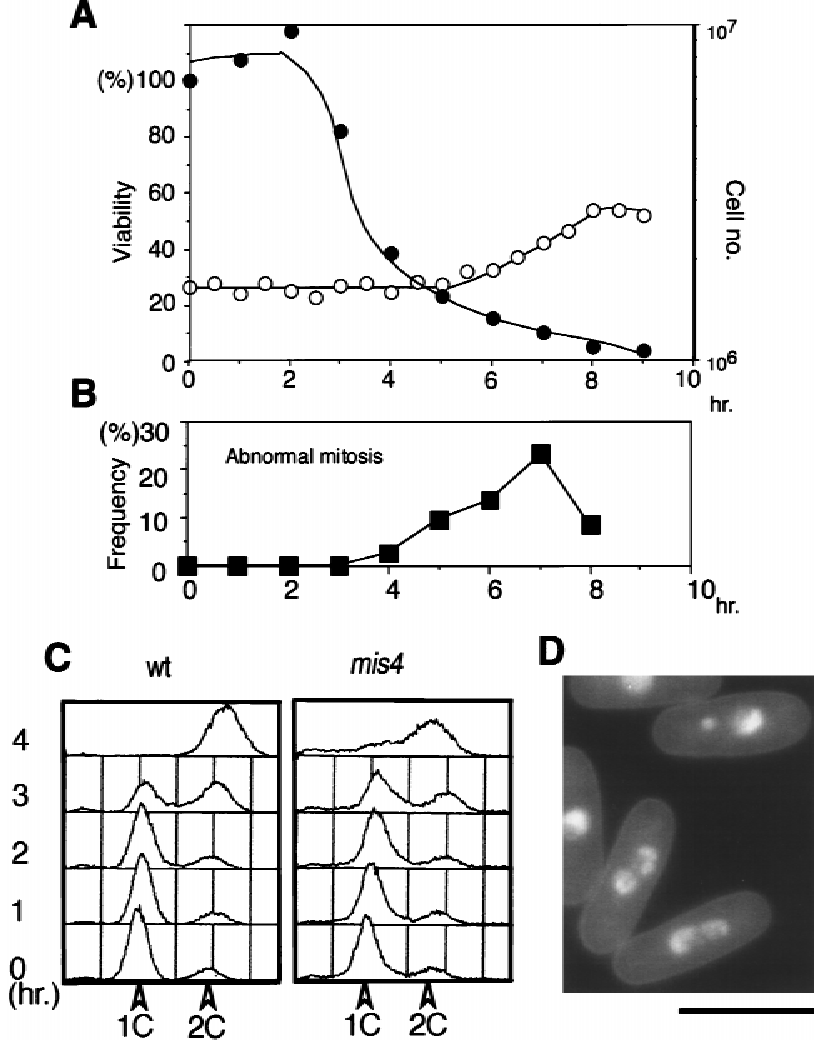

Figure 2. A lethal event occurs in S phase when mis4-242 mutant cells are released from $\mathrm{G}_{1}$ arrest. Mutant cells were nitrogen source-starved at $26^{\circ} \mathrm{C}$ to arrest then in $\mathrm{G}_{1}$. Cells were then released in complete medium at $36^{\circ} \mathrm{C}$. (A) Cell viability (0; decreasing from 3 to $4 \mathrm{hr}$ after the shift) and cell number $(O$; increasing around $8-9 \mathrm{hr})$. (B) Frequency of cells showing unequal chromosome segregation (peaking at $7 \mathrm{hr}) .(C)$ DNA content measured by FACScan analysis showed that DNA replication in mis 4-242 cells took place around 3-4 hr, coinciding with the occurrence of cell inviability. (wt) Wild-type control. $(D)$ Examples of mutant cells showing abnormal anaphase. Cells stained by DAPI. Bar, $10 \mu \mathrm{m}$.

presence of the disrupted gene in the genomes of heterozygous diploids was verified by genomic Southern hybridization (data not shown). Tetrad analysis of the sporulated diploids indicated that only two spores were viable and that all viable spores were nondisrupted Ura ${ }^{-}$. The $\mathrm{mis}^{+}$gene was therefore absolutely required for viability. The mis4-disrupted cells were observed after DAPI staining, chromosome condensation was observed in mitosis, and missegregation phenotypes resembling mis4-242 were seen (data not shown).

\section{HU-sensitive mis4-242 maintains replication checkpoint control}

mis4-242 was found to be hypersensitive to $\mathrm{HU}$; like cdc2-3w it did not form colonies in $5 \mathrm{~mm} \mathrm{HU}$ at $26^{\circ} \mathrm{C}$ (Fig. 4A). Mutant cells cultured at $26^{\circ} \mathrm{C}$ in liquid medium containing $15 \mathrm{~mm} \mathrm{HU}$ were greatly elongated (Fig.
4C), although they contained a 1C DNA content determined by FACS analysis (Fig. 4B), showing that HU-induced replication checkpoint control, which restrained entry into mitosis, was maintained in the mutant. Note that cell viability did not decrease under these conditions for up to $8 \mathrm{hr}$ (Fig. 4D).

The HU-sensitive phenotype of the mutant was further examined at $36^{\circ} \mathrm{C}$. Mutant cells initially arrested in $\mathrm{G}_{1}$ at $26^{\circ} \mathrm{C}$ were released in rich medium at $36^{\circ} \mathrm{C}$ either in the presence or the absence of HU. These cells contained 1C DNA even after $7 \mathrm{hr}$ in the presence of HU whereas cells without HU completed S-phase at $4 \mathrm{hr}$ (Fig. 4E). Cells were highly elongated in the presence of HU for $7 \mathrm{hr}$ at $36^{\circ} \mathrm{C}$ (Fig. 4F), indicating that replication checkpoint control remained in the mutant even at that temperature. Cell viability in the presence of $\mathrm{HU}$ remained high until $4 \mathrm{hr}$ but then decreased and reached $10 \%$ after $6 \mathrm{hr}$ (Fig. 4G). This timing of inviability was 1-2 hr slower than that of mutant cells that lost viability during the progression through $S$ phase in the absence of HU. Notably, however, most mutant cell nuclei after 6 $\mathrm{hr}$ in the presence of HU contained only a 1C DNA content. The prolonged S-phase block by HU in mis4-242 thus caused severe cell lethality.

\section{mis4-242 cells are sensitive to UV irradiation}

In addition to HU-sensitivity, mis4-242 cells were UVirradiation sensitive at $26^{\circ} \mathrm{C}$ (Fig. 5A). To determine whether the DNA damage checkpoint control was retained after irradiation, the frequency of septum formation after damage was measured. Wild-type cells treated with UV irradiation showed a rapid decrease in the septum index as a result of the great delay for mitotic entry (Fig. 5B, left). Cells were elongated with a single nucleus (data not shown). The septation index was also reduced in mis4-242 mutants after irradiation (right panel). The recovery of this index, however, was delayed (the mutant took $2-3 \mathrm{hr}$, rather than $1.5 \mathrm{hr}$ as shown by the wild type). These results show that mis4-242 mutants retain damage-sensing checkpoint control, but appear to be impaired in the process of repair or recovery.

mis4 is synthetic lethal with a DNA ligase mutation at $26^{\circ} \mathrm{C}$

We looked for mutations that interacted with mis4-242. For this purpose, a number of genetic crossings were done between mis4-242 and various cut or cdc mutations, and the phenotypes were tested. A strong interaction with the cdc17 mutation, which is defective in DNA ligase, was found. The mis4-cdc17 double mutant did not form colonies at $26^{\circ} \mathrm{C}$, but formed small colonies at $22^{\circ} \mathrm{C}$ (Fig. 5C). Weak interactions were found for other double mutants with mis5, cdc20, or cdc22, all defective in replication; the double mutants failed to form colonies below the restrictive temperature of single mutants. 
Figure 3. The gene product of $\mathrm{mis}^{+}$is similar to that of $C$. cinereus Rad9 and proteins present in yeast and mammals. (A) Isolation of plasmid carrying the mis $4^{+}$ gene. The temperature-sensitive phenotype of mis4-242 was rescued (indicated by + ) by pKT221 and some of its subclones. (Arrow) Coding region; (vertical white lines) introns. pKF201 is the minimal functional clone that contains the whole coding region. (B) Schematic representation of S. pombe Mis4, S. cerevisiae Scc2, and C. cinereus Rad9. Partial sequences derived from human and mouse cDNAs are also shown. The conserved regions ( $22 \%$ to $38 \%$ identity) are hatched. Mouse AA062272 and human HMHBC4244 sequences are similar to the carboxyl termini of Mis4/Scc2/Rad9. The DDBJ accession number for the nucleotide sequences of Mis4 is AB016866. (C) Immunoblot of $S$. pombe extracts containing Mis4-HA inte-
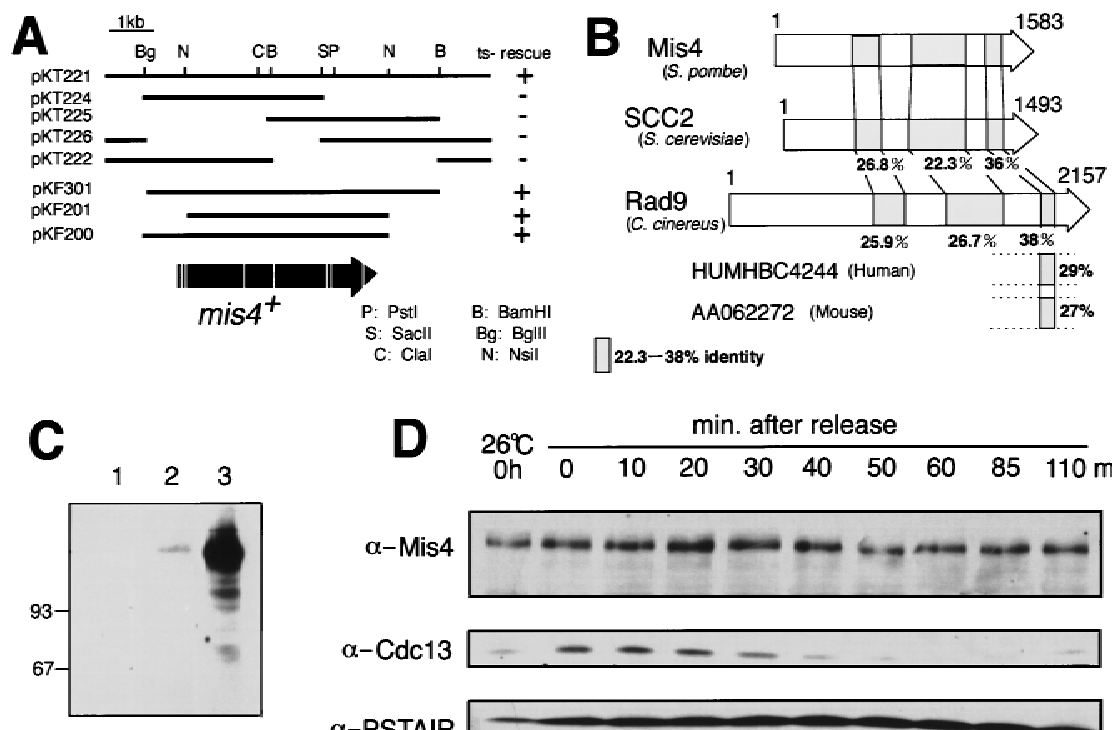
grated into the chromosome (lane 2) or a plasmid containing Mis4-HA (lane 3). (lane 1) Control extracts of cells without integration and carrying the vector. (D) The immunoblot patterns of cdc25 mutant block and release experiments, showing the levels of Mis4, Cdc2 (PSTAIR), and mitotic cyclin (Cdc13). Cyclin destruction occurred $30 \mathrm{~min}$ after the shift to the permissive temperature $\left(26^{\circ} \mathrm{C}\right)$. The Mis 4 protein band slightly increased its mobility after $50 \mathrm{~min}$ (when Cdc13 levels dropped), but this was not reproducible.

Hypercondensation of chromosome occurs, but whole sister chromatids are separated in metaphase-arrested mis4-242 cells

Incorrect chromosome segregation in mis4 mutants strongly suggested that Mis4 may act to hold sister chromatids together until anaphase. Therefore, we examined the phenotypes of mitotically arrested mis 4 mutants by introducing a second mutation that blocked cells in metaphase. Mis4 sister chromatids were condensed and held together in metaphase-arrested cut 9 mutant cells; Cut9 is an essential component of the 20S APC/cyclosome complex required for anaphase-promoting proteolysis (Yamada et al. 1997). The double mutant cut9mis4 was constructed and examined. Single cut 9 and double cut $9-$ mis 4 mutants were arrested in $\mathrm{G}_{1}$ by nitrogen starvation and then released in rich medium at $36^{\circ} \mathrm{C}$. In both mutant strains, $\mathrm{Cdc} 2$ protein kinase activity was measured and found to be high for 5-8 hr (Fig. 6A), and neither Cut2 nor Cdc13 was degraded (Fig. 6B). In single cut9 mutants at $36^{\circ} \mathrm{C}$, chromosomes were highly condensed (Samejima and Yanagida 1994) whereas in the double cut9-mis4 mutants, they were also highly condensed but scattered (Fig. 6C). mis4-242 thus differs from budding yeast $m c d 1-1$, which was shown to be defective in chromosome condensation (Guacci et al. 1997).

To show that sister chromatids were separated in double mutant cells, FISH analysis (Uzawa and Yanagida 1992; Funabiki et al. 1993) was performed with three kinds of probes, namely, sequences of rDNA located on chromosome III, unique sequences derived from the centromere II (cen II), and the telomere of the short arm of chromosome II (tel II). As seen in a typical example of cells in Figure 6D-F, the regions of rDNA, centromeres, and telomeres were all separated in double mutant cells, but never in single cut9 mutants. These results definitively demonstrate that Mis4 is needed to associate all the regions of sister chromatids in metaphase-arrested cells.

\section{Localization of Mis4 to the nuclear chromatin region}

The results described above suggest that Mis4 is a nuclear chromosomal protein. Mis4 is indeed localized to the nucleus. The single copy gene of $\mathrm{mis}^{+}$was tagged with jellyfish green fluorescent protein (GFP) and integrated into the chromosome (see Materials and Methods). The carboxyl terminus of Mis4 was tagged with GFP and the promoter was kept native. This construct was functional, as cells substituting the temperaturesensitive mutant gene with the GFP construct could grow normally. As shown in Figure 6G, the nuclear chromatin in cells expressing GFP-Mis4 was fluorescent with intense nuclear punctate signals. Note that fluorescence signals are enriched in rDNA (Uzawa and Yanagida 1992) as well as other restricted chromatin regions. No decrease of the signals was detected during mitosis. Punctate GFP signal was also observed in anaphase nuclear chromatin. In nda3 mutant cells expressing Mis4-GFP, the signals were localized to condensed chromosomes (Fig. 6H). Some of the chromosomal punctate signals were also seen. Mis4, at least its fraction, was associated with chromosomes throughout the cell cycle.

Premature sister chromatid separation occurs in mis4-242 cells during the post-replicative interphase A question was raised whether sister chromatids were 


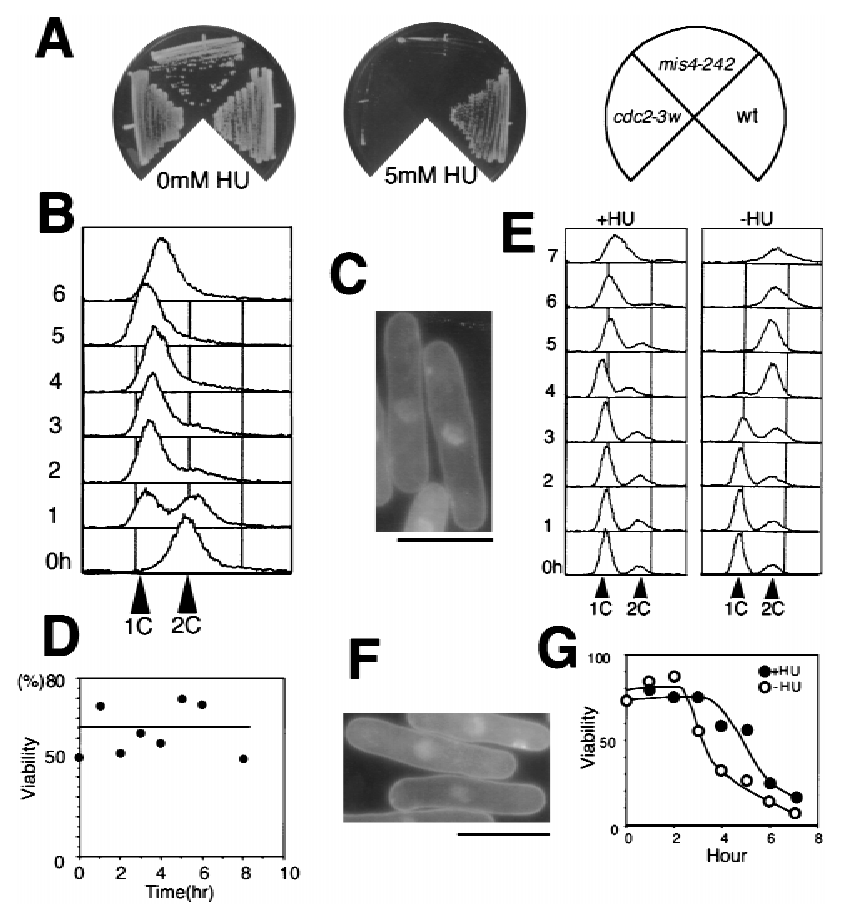

Figure 4. Mis4 is sensitive to $\mathrm{HU}$ and becomes lethal in the S-phase block induced by HU. (A) like Cdc2-3w cells mis4-242 cells failed to form colonies in the presence of $5 \mathrm{~mm} \mathrm{HU}$ at $26^{\circ} \mathrm{C}$. (B) FACS analysis indicating that replication was blocked (DNA content is 1C) for $6 \mathrm{hr}$ in mis4-242 in the presence of $15 \mathrm{~mm} \mathrm{HU}$ at $26^{\circ} \mathrm{C}$. (C) Mutant cells elongated with the interphase single nucleus. Bar, $10 \mu \mathrm{m}$. (D) Viability of mutant cells blocked by 15 mM HU remained high for $6 \mathrm{hr}$ at $26^{\circ} \mathrm{C}$. $(E-G)$ Mutant cells were nitrogen starved to cause arrest in $\mathrm{G}_{1}$ at $26^{\circ} \mathrm{C}$ and then released in complete medium at $36^{\circ} \mathrm{C}$ in the presence or the absence of $15 \mathrm{~mm}$ HU. $(E)$ The DNA content of mutant cells remained 1C for $7 \mathrm{hr}$ in the presence of HU but increased to $2 \mathrm{C}$ after $4 \mathrm{hr}$ in the absence of HU. $(F)$ Mutant cells were elongated at $36^{\circ} \mathrm{C}$ in the presence of HU. $(G)$ Viability of mutant cells decreased to $50 \%$ and $20 \%$ after 3 and $5 \mathrm{hr}$, respectively, in the absence of HU (O), whereas viability decreased to $50 \%$ and $20 \%$, respectively, after 5 and $7 \mathrm{hr}$ in the presence of $\mathrm{HU}(\mathbf{O})$. Mutant cells became inviable while the DNA content was still 1C.

prematurely separated in mis4-242 interphase cells after replication. The mutant cells were initially arrested in $\mathrm{G}_{1}$ by nitrogen starvation and then released in complete medium at $36^{\circ} \mathrm{C}$. DNA replication occurred about $4 \mathrm{hr}$ after the shift (Fig. 7A). Mitosis took place around $7 \mathrm{hr}$ (see Fig. 2). Cells were collected at 2, 4, and $6 \mathrm{hr}$, and to visualize behavior of the centromere DNA of chromosome I (cen1) in living cells (see Materials and Methods; Straight et al. 1996; Nabeshima et al. 1998) the cen1GFP technique was applied to score the frequencies of cells revealing the two separated signals of the unique cen 1 DNA. A large fraction of interphase cells showed the two split cen 1 signals after 4-6 hr ( 30\% and 40\% after 4 and $6 \mathrm{hr}$, respectively). Examples of cells showing the split cen1-GFP dots in interphase are shown in Figure $7 \mathrm{C}$. Hence, we concluded that the sister chromatids were prematurely separated after the S phase and continued to be split until the following mitosis in mis 4 mutant cells. Note that these interphase cells revealing the two split signals did not contain the spindle at all, so that separation was not attributable to the spindle force. The decrease of viability coincided with the increase of split signals, suggesting that the cause for the loss of viability was the failure to form the sister chromatid cohesion.

\section{Viability of metaphase-arrested mis4 mutant cells is quickly lost}

As $S$ phase in growing fission yeast cells was initiated immediately after mitotic anaphase, we wanted to assure that mis 4 cells truely lost viability during the first mitosis when cells were shifted from early $\mathrm{G}_{2}$ (see Fig. 1). For this purpose, viability of the double mutants cut9mis4 and cut7-mis4 was measured and compared with that of single mutants (cut7 mutant was arrested at prophase; Hagan and Yanagida 1990). Viability of the double mutants, which were cultured from $\mathrm{G}_{2}$ and arrested at metaphase at $36^{\circ} \mathrm{C}$, decreased more rapidly (reduced to $10 \%-15 \%$ after $2 \mathrm{hr}$ ) than single cut 9 and cut 7 mutants $(60 \%-70 \%)$, suggesting that Mis4 was needed to maintain viability in mitotically arrested cells. The viability of double mutant cdc25-mis4 cells (similarly cultured as described above but arrested in late $\left.G_{2}\right)$, on the other hand, decreased much more slowly (60\% after $2 \mathrm{hr}$ ) than single mis 4 mutants $(30 \%)$, indicating that viability is lost in mis 4 mutant much faster in metaphase- rather than in $\mathrm{G}_{2}$-arrested cells.

\section{Discussion}

This study describes results demonstrating that fission yeast Mis4 is required for sister chromatid cohesion, its loss leading to premature sister chromatid separation. Properties of Mis4 differ from those of the previously identified $\operatorname{Rad} 21 / \operatorname{Mcd} 1 \mathrm{p} / \mathrm{Scc} 1 \mathrm{p}$ cohesin. In Xenopus, XRAD21 forms a complex with the two SMC subunits (XSMC1 and XSMC3) and two other unknown subunits, p155 and p95 (Losada et al. 1998). This five-member Xenopus cohesin complex is required for sister chromatid cohesion: Immunodepletion of the complex causes defects in sister chromatid cohesion in subsequent mitosis. In S. pombe, besides Rad21 (Birkenbihl and Subramani 1992), proteins highly similar to Smc1 and Smc3 are present, and their complex products were obtained (T. Sutani, J. Morishita, T. Tomonaga, M. Yanagida, unpubl.). Mis4 does not seem to be one of the unknown subunits in the cohesin complex. Immunoprecipitation with antibodies against Smc3 or Rad21 did not coprecipitate Mis4 (K. Furuya, T. Sutani and M. Yanagida, unpubl.). In other experiments with anti-Cut3 antibodies, Mis4 was not coprecipitated with Cut3, indicating that Mis4 is the subunit of neither cohesin nor condensin. Mis4 may exist in an oligomeric complex, as it sediments at $\sim 10 \mathrm{~S}$ in sucrose gradient centrifugation (K. Furuya, K. Takahashi, and M. Yanagida, unpubl.), distinct from the $14 S$ peak of the five-member cohesin. 
Furuya et al.
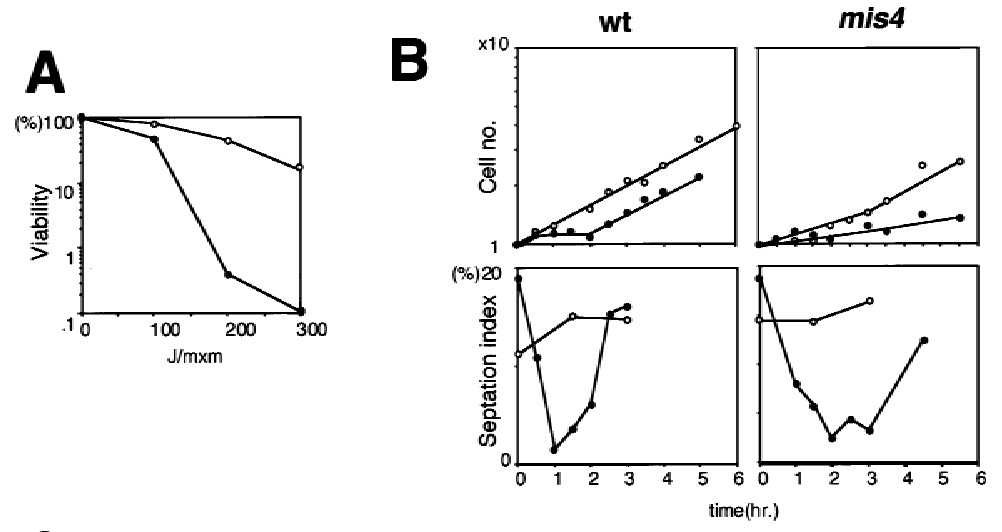

Figure 5. 5 UV sensitivity and instability of chromosomes in mis4-242. (A) mis4242 cells are $(0)$ sensitive to UV irradiation at $26^{\circ} \mathrm{C}$. (O) Wild-type control. (B) The septation indices of wild-type and mis4242 cells were equally reduced by 100 joules of UV irradiation at $26^{\circ} \mathrm{C}(\mathbf{\bullet})$, but the recovery of the septation index to the level of normal growing cells was significantly delayed in mutant cells $(O) 0$ joules of UV irradiation. $(C)$ The double mutant mis4-242-cdc17-K42 did not form colonies even at $26^{\circ} \mathrm{C}$.
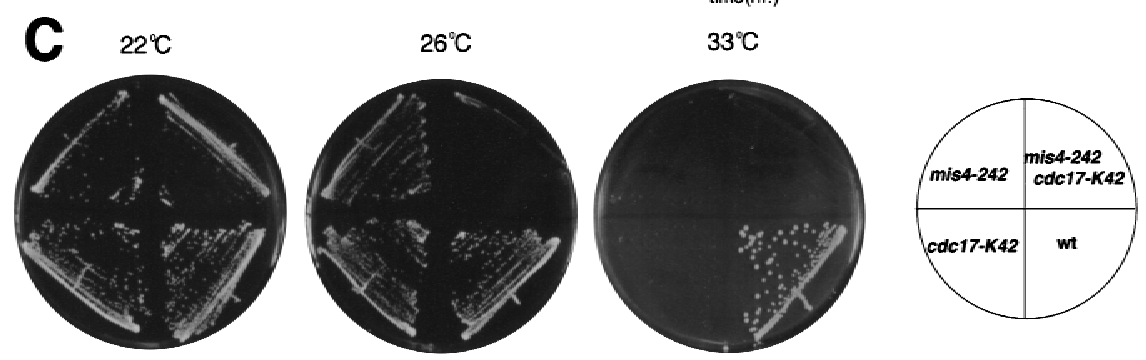

Properties of Mis4 suggest that the manner in which Mis4 is involved in sister chromatid cohesion is distinct from $\operatorname{Rad} 21 / \operatorname{Scc} 1 / \operatorname{Mcd} 1$, the subunit of cohesin. First, Mis4 is not directly related to condensation whereas Mcdl is shown to be required for chromosome condensation (Guacci et al. 1997). However, the requirement of cohesin (Mcdlp) for chromosome condensation has recently been challenged in a Xenopus system by Losada et al. (1998). Second, Mis4 is not the target of the APC/ cyclosome whereas Mcd1/Scc1 requires the APC/cyclosome for its destruction (Michaelis et al. 1997). Mis4 is present in anaphase- and $\mathrm{G}_{1}$-arrested cells; Mis4-GFP signals were clearly visualized in anaphase chromosomes. Third, Mis4 is required to maintain viability during the S phase, though replication per se is not blocked in mis4 mutants. Synthetic lethality with a DNA ligase mutant suggests that gaps or nicks, causing chromosome instability or recombination, might be produced in mis 4 mutant cells. UV and HU hypersensitivity also suggest the presence of chromosomal defects generated by mis 4 mutation. During ongoing replication and $\mathrm{G}_{2}$, correction of damage on one sister is normally facilitated by the close proximity of the undamaged sister, but loss of cohesion interferes with such interactions. Recombinational repair might also be defective in mis4 mutant as the sisters are farther away. But these possibilities remain to be determined. Taken together, we conclude that Mis4 belongs to a distinct class of proteins required for sister chromatid cohesion. It functions differently from $\operatorname{Rad} 21 / \operatorname{Scc} 1 / \mathrm{Mcd} 1$, and is unrelated to the amino acid sequences of SMC proteins. Mis4, together with $C$. cinereus Rad9, budding yeast Scc2, mouse AA062272, and human HUMHBC4244 may constitute a novel family of sister chromatid adhesion molecules. We propose naming Rad9/Scc2/Mis4 adherin to distinguish it from

the original SMC-interacting cohesin. We discuss possible roles of Mis4 for sister chromatid cohesion, ad propose a simple model.

\section{Mis4 is an essential component of sister chromatid cohesion}

Punctate nuclear signals of Mis4-GFP suggest that Mis4 may preferentially associate with distinct chromosomal sites. In mitotically arrested cells, the dotted images were also seen on hypercondensed chromosomes. The punctate signals might colocalize with repetitive regions such as rDNA. As the rDNA repeats are located at the ends of chromosome III, Mis4 may be enriched in other repetitive chromosomal ends. Mis4 did not appear to be enriched in the centromeric regions, however. Mis4 may recognize DNA sequence motifs or protein(s) enriched in such particular chromosomal regions. Further investigation is needed to precisely identify Mis4 localization, however. The conserved carboxy-terminal regions in Rad9/Scc2/Mis4 adherin molecules form no nucleotide binding sequence nor known DNA-binding motif, and their molecular function is unknown. Three Cdc2 kinase phosphorylation sites are present in the amino termini of Mis4 and Rad9, but not in Scc2. The amino-terminal region may have a regulatory function, as that of Mis4 also contains the nuclear localization signal (Y. Toyoda, K. Furuya, and M. Yanagida, unpubl.).

We show that whole chromatids are separated in metaphase-arrested mis4 mutant cells using three different hybridization probes for the FISH analysis. Premature sister chromatid separation in mis 4 mutants is also demonstrated in interphase $G_{2}$ cells by inactivation of mutant Mis 4 protein in $G_{1}$. Separation is not caused by the spindle force, as $G_{2}$ cells in fission yeast are completely 


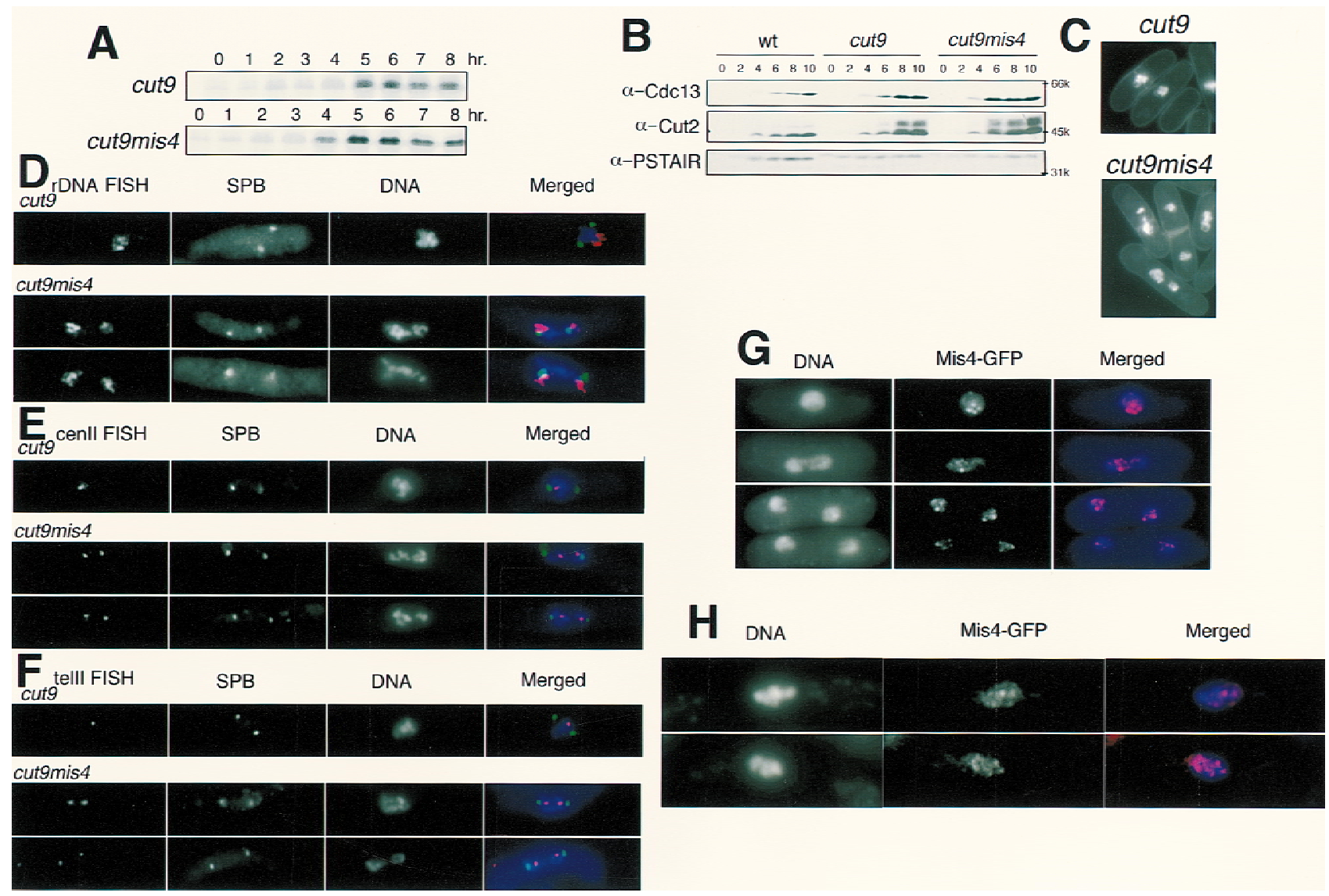

Figure 6. Sister chromatid separation occurs in metaphase-arrested mis4-242 cells. (A) Single cut9 and double cut9-mis 4 mutant cells initially arrested in $\mathrm{G}_{1}$ by nitrogen starvation and then released in complete medium and cultured at $36^{\circ} \mathrm{C}$ for $8 \mathrm{hr}$. The $\mathrm{H} 1 \mathrm{kinase}$ activity was high in both single cut9 and double cut9-mis 4 mutant cells. (B) Cut2 and Cdc13 proteolysis was absent. (C) DAPI-stained single cut9 and double cut9-mis4 mutant cells. Both mutant strains were arrested at metaphase with condensed chromosomes. $(D-F)$ FISH analysis applied to arrested single cut9 and double cut9-mis4 cells with probes from rDNA $(D)$ the unique centromere region cenII $(E)$, and the unique telomere region telII $(F)$. Counter staining by anti-SPB antibody or DAPI is also shown with the merged image. Bar, $10 \mu \mathrm{m}$. $(G)$ Localization of Mis4-GFP in the nucleus. The mis $4^{+}$gene was tagged with GFP and integrated into the chromosome as a single-copy gene with the native promoter. In living cells, the GFP signal was seen in nuclear chromatin. Signals were seen in the nucleus throughout the cell cycle. Punctate nuclear signals were also seen, which appeared to localize to rDNA and telomeres. $(H)$ Mis4-GFP signals were seen on condensed chromosomes produced in the mitotically arrested $\beta$-tubulin mutant nda3-311 at $20^{\circ} \mathrm{C}$.

devoid of spindles. Great reduction in the level of Mis4 protein in mutant cells probably causes the loss of connection between sister chromatids and results in the premature separation phenotype. Mis4 may thus provide a direct link between chromatids or may alter chromatids so that they have a structure capable of interacting with cohesin. No genetic interaction, however, has been found between mis 4 and rad21 mutations so far. It is of considerable interest to determine whether these two chromatid cohesion molecules are functionally interrelated.

\section{Requirement for Mis4 in S phase and M phase}

When mis 4 mutants had been inactivated in $\mathrm{G}_{1}$ or $\mathrm{G}_{2}$, a cell cycle defect was produced in the subsequent $S$ or $M$ phase, respectively, suggesting that Mis4 was required for both these phases. Viability was kept to be relatively high in the gap stages between $S$ phase and M phase. The failure to form sister chromatid cohesion is probably the cause of mutant cell death during S phase; this defect could not be restored when cells had passed this phase. The requirement for Mis4 in M phase is intriguing, as the progression of the first mitosis is an unrecoverable lethal event but cytologically indistinguishable from normal mitosis. One explanation of this phenotype is that mutant cells could not establish, during the first mitosis, a particular chromosome conformation that is specifically required for the reforming of sister chromatid cohesion in the subsequent $\mathrm{S}$ phase. The major chromosome reorganization reported to take place at the onset of mitosis in vertebrate cells (Losada et al. 1988) may also take place in S. pombe. The lack of Mis4 in the reorganization step may not affect sister chromatid separation immediately thereafter but may lead to the loss of the ability to reform cohesion in the subsequent $\mathrm{S}$ phase. If sister cohesion is not made in $\mathrm{S}$ phase, premature sister chromatid separation inevitably occurs in $\mathrm{G}_{2}$ and gives 
Figure 7. Sister centromeres are prematurely separated in the interphase state of mis4-242 after replication. The cen1 DNA was visualized by use of the LacI system; (Straight et al. 1996). Lac repressor was tagged with GFP and an NLS and expressed in a $S$. pombe strain containing LacO repeats integrated near cen 1 (Nabeshima et al. 1998) mis4-242 cells (and a wild-type control) expressing the LacI-NLS-GFP and integrated with the LacO repeat were arrested in $\mathrm{G}_{1}$ by nitrogen starvation at $26^{\circ} \mathrm{C}$, shifted to complete medium, and cultured at $36^{\circ} \mathrm{C}$ for $8 \mathrm{hr}$. (A) FACS analysis indicating that replication occurred in mis4-242 cells after $4 \mathrm{hr}$ and was completed before $6 \mathrm{hr}$ as in the wild type (not shown). (B) cenl-GFP signals were visualized, and the frequencies of cells revealing two signals $(O)$ graphed with the frequencies of viable cells $(\bullet)$. Because mitotic cells started to increase after $7 \mathrm{hr}$, data for the two split signals were not taken after that time. (C) Example of mis4-242 cells showing the two split cen 1 signals. The wild-type control is also shown. Bar, $10 \mu \mathrm{m}$.
A

B
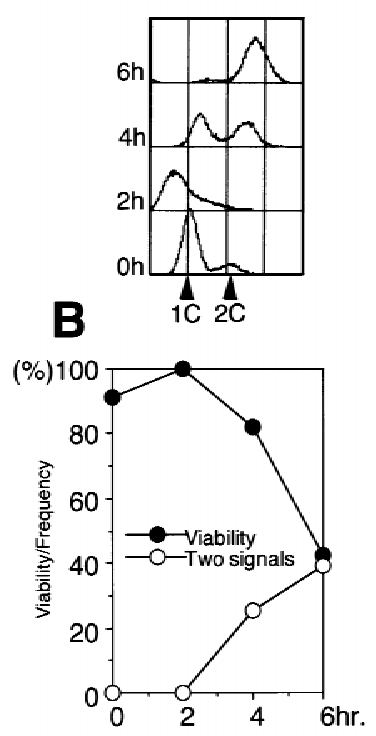

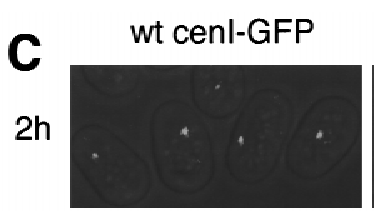

mis4 cenl-GFP

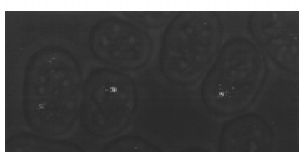

$4 \mathrm{~h}$
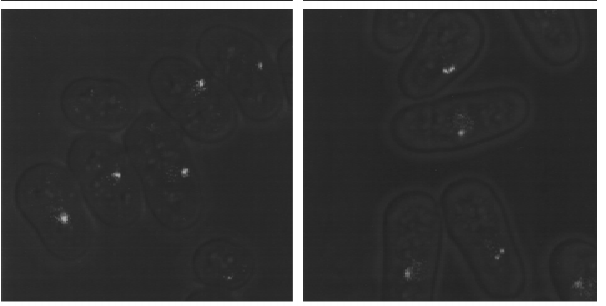

$6 \mathrm{~h}$
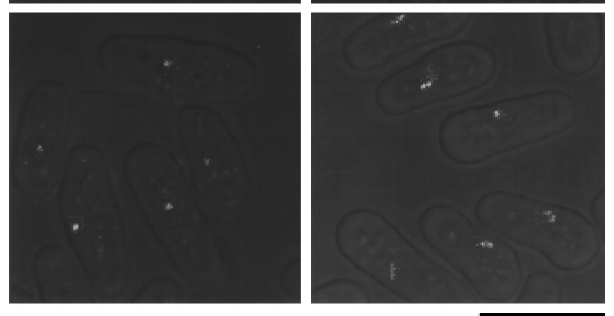

rise to nearly random chromosome segregation in the following mitosis. Bi-orientation of sister centromeres will not be achieved in metaphase if sister chromatid cohesion is entirely abolished. A similar loss of cohesion, in the restricted region, was reported for the mutant of Mis6, a centromere-specific connector protein essential for association of sister centromeres that acts during the $G_{1} / S$ phase (Saitoh et al. 1997).

\section{Mis4 and the onset of anaphase}

The removal of cohesin from chromosomes upon entry into anaphase requires Esp1, which is essential for sister chromatid separation (Ciosk et al. 1998). Anaphase-promoting proteolysis triggers the destruction of Pds1, an inhibitor of Esp1, resulting in the activation of Esp1, which may subsequently promote disruption of the linkage between chromatids by removing Scc1. However, we have not yet found any genetic and biochemical relations between Mis4 and Cut1 (a homolog of Esp1) or between Mis4 and Cut2 (a homolog of Pds1; Kumada et al. 1998). Therefore, we consider a different mechanism for the implication of Mis4 in the onset of anaphase.

We propose a simple model in which Mis4 associates with chromosomes throughout the cell cycle and forms the connection between sister chromatids directly or indirectly through another linking protein. Disruption of the link might occur by splitting of the oligomeric Mis4 complex into halves or removal of the linking protein from chromosomes in anaphase while Mis4 remains associated with chromosomes. Post-translational modification such as protein dephosphorylation (Ohkura et al. 1989) may be important in disconnecting the linkage. Many other models may be possible, but the one described here is among the simplest. During S phase, Mis4 should be added to a newly replicated DNA. If Mis4 is inactive at the time of replication, the connection between chromatids will not be made, resulting in precocious separation of sister chromatids after replication. Once formed, sister cohesion would be maintained even if Mis4 is later inactivated, but would result in normallooking mitosis with a cryptic irreversible defect. Identification of actual chromosome defects at the molecular level will be an important step toward understanding the regulation of sister chromatid cohesion. Both cohesin and adherin are essential for sister chromatid cohesion, and the loss of either one results in premature chromatid separation. Determination of their hierarchical relationship, if any exists, will also be a subject for future investigation.

\section{Materials and methods}

Strains and media

S. pombe haploid strains were used. Handling of temperaturesensitive strains, mis4, cut9, cdc25, and $c d c 17$ and media used were described previously (Takahashi et al. 1994; Yamada et al. 1997). The strain used for integration of the cloned mis $4^{+}$gene onto the chromosome was $\mathrm{h}^{-}$mis4-242 ura4.

\section{Plasmids}

Procedures for gene cloning in $S$. pombe by transformation were described previously (Takahashi et al. 1994). An S. pombe genomic library was used for isolation of the mis $4^{+}$gene. The selection marker was the $S$. cerevisiae LEU2 gene. Integration plasmid pYC6 containing the $\mathrm{ura}^{+}$gene was used for integration of a $3-\mathrm{kB}$ BamHI-BglII fragment into the chromosome by homologous recombination. The carboxyl terminus of mis $4^{+}$ was tagged in frame with the $3 \times$ HA antigen. A plasmid carrying the tagged mis $^{+}$was able to suppress the temperature-sensitive phenotype of mis4-242. The vector plasmid used was pSK248. 


\section{Molecular biological methods}

The nucleotide sequence of a 7-kb long BamHI-BgIII fragment in pKT221 was determined. Three of the presumed introns in the carboxyl terminus were confirmed by PCR amplification of cDNA. 12CA5, an antibody against HA, was used for immunoblotting to detect Mis4-HA. Cells were irradiated at $36^{\circ} \mathrm{C}$ in a warm room with temperature control. The FACScan analysis was performed according to the procedures described by Costello et al. (1986). Cells collected were washed in 70\% ethanol. DNA contents were measured by a Becton-Dickinson FACScan apparatus after propidium iodide staining.

\section{Gene disruption}

The procedures described by Rothstein (1983) were followed. The BgIII-PstI fragment in pKT221 was subcloned, and an internal HindIII fragment was removed and substituted with the S. pombe $\mathrm{ura4}^{+}$gene. The resulting plasmid, pKF230, was digested with SnaBI and SpeBI and used for transformation of a diploid (5A/1D). Stable transformants for the $\mathrm{Ura}^{+}$marker were isolated, and gene disruption was verified by genomic Southern hybridization. The resulting heterozygous diploid $\Delta 10$ was sporulated and tetrads dissected.

\section{Synchronous culture}

The procedures described by Moreno et al. (1989) and Kinoshita et al. (1990) were followed. A Beckman elutriator rotor was used for collecting small $G_{2}$ cells. Procedures for nitrogen starvation were employed to concentrate cells in $G_{1}$ followed by release into complete medium (Saka and Yanagida 1993). EMM2-N lacking $\mathrm{NH}_{4} \mathrm{Cl}$ was used for starvation $\left(2 \times 10^{7} / \mathrm{ml}\right.$ for $24 \mathrm{hr}$ at $26^{\circ} \mathrm{C}$ ). A cell concentration of $1 \times 10^{6} / \mathrm{ml}$ was employed for the shift into complete medium at $36^{\circ} \mathrm{C}$. Block and release experiments with the $c d c 25$ mutant were done as described (Funabiki et al. 1996; Yamada et al. 1997).

\section{GFP constructs}

The use of GFP for tagging the carboxyl terminus of Mis4 and integrating of the construct into the chromosome was done as described (Nabeshima et al. 1995). The integration vector pYC11 was used, and the resulting plasmid pMIS4-GFP was used for transformation of the strain $h^{-}$leu1 mis4-242 after linearization. The resulting $\mathrm{Leu}^{+} \mathrm{Ts}^{+}$integrants were used for microscopy. A fission yeast strain was constructed to visualize cenDNA (Nabeshima et al. 1998) A haploid S. pombe strain $h^{-}$ lys1 his 7 was transformed simultaneously with the two plasmids pMK24A and pMK2A. pMK24A carried the GFP-LacINLS (Straight et al. 1996) driven by the promoter region of dis $1^{+}$ (Nabeshima et al. 1995) and the his $7^{+}$gene; NLS is the nuclear location signal. The plasmid pMK2A contained the LacO repeat and the lys $1^{+}$gene, which is tightly linked to the cen 1 locus $(\sim 30$ kb; Takahashi et al. 1992). The resulting $\mathrm{Lys}^{+} \mathrm{His}^{+}$transformants were isolated. Transformant MKY5A contained the integrated GFP-LacI-NLS gene at the his 7 locus and also the LacO array at the lys1 locus.

\section{Microscopy}

FISH analyses and immunofluorescence microscopy with antitubulin antibody were done as described by Funabiki et al. (1993). Staining of DNA in living cells was done as described previously (Chikashige et al. 1994). Detailed procedures for the culture of cells for microscopy were described by Nabeshima et al. (1997). A MRC-1024 confocal microscope was used with excitation at $488 \mathrm{~nm}$ and the filter 522DF32.

\section{Acknowledgments}

This study was supported by grants from the Japan Science Technology Corporation, the Human Frontier Science Program Organization, and the Science and Technology Agency of Japan.

The publication costs of this article were defrayed in part by payment of page charges. This article must therefore be hereby marked 'advertisement' in accordance with 18 USC section 1734 solely to indicate this fact.

\section{References}

Adachi, Y., J. Usukura, and M. Yanagida. 1997. A globular complex formation by $\mathrm{Ndal}$ and the other five members of the MCM protein family in fission yeast. Genes to Cells 2: 467479.

Birkenbihl, R.P. and S. Subramani. 1992. Cloning and characterization of rad21 an essential gene of Schizosaccharomyces pombe involved in DNA double-strand-break repair. Nucleic Acids Res. 20: 6605-6611.

Chikashige, Y., D.-Q. Ding, H. Funabiki, T. Haraguchi, S. Mashiko, M. Yanagida, and Y. Hiraoka. 1994. Telomere-led premeiotic chromosome movement in fission yeast. Science 264: $270-273$.

Ciosk, R., W. Zachariae, C. Michaelis, A. Shevchenko, M. Mann, and K. Nasmyth. 1998. An ESP1/PDS1 complex regulates loss of sister chromatid cohesion at the metaphase to anaphase transition in yeast. Cell 93: 1067-1076.

Costello, G., L. Rodgers, and D. Beach. 1986. Fission yeast enters the stationary phase G0 state from either mitotic G1 or G2. Curr. Genet. 11: 119-125.

Funabiki, H., I. Hagan, S. Uzawa, and M. Yanagida. 1993. Cell cycle-dependent specific positioning and clustering of centromeres and telomeres in fission yeast. I. Cell Biol. 121: 961-976.

Funabiki, H., H. Yamano, K. Kumada, K. Nagao, T. Hunt, and M. Yanagida. 1996. Cut2 proteolysis required for sister-chromatid separation in fission yeast. Nature 381: 438-441.

Guacci, V., D. Koshland, and A.V. Strunnikov. 1997. A direct link between sister chromatid cohesion and chromosome condensation revealed through the analysis of MCD1 in $S$. cerevisiae. Cell 91: 47-57.

Hagan, I.M. and M. Yanagida. 1990. Novel potential mitotic motor protein encoded by the fission yeast $\mathrm{cut}^{+}$gene. $\mathrm{Na}$ ture 347: 563-566.

Hirano, T., S. Funahashi, T. Uemura, and M. Yanagida. 1986. Isolation and characterization of Schizosaccharomyces pombe cut mutants that block nuclear division but not cytokinesis. EMBO J. 5: 2973-2979.

Holloway, S., M. Glotzer, R.W. King, and A.W. Murray. 1993. Anaphase is initiated by proteolysis rather than by the inactivation of maturation-promoting factor. Cell 73: 13931402.

Kerrebrock, A.W., D.P. Moore, J.S. Wu, and T.L. Orr-Weaver. 1995. Mei-S332, a Drosophila protein required for sisterchromatid cohesion, can localize to meiotic centromere regions. Cell 83: 247-256.

Kinoshita, N., H. Ohkura, and M. Yanagida. 1990. Distinct, es- 
Furuya et al.

sential roles of type 1 and $2 \mathrm{~A}$ protein phosphatases in the control of the fission yeast cell division cycle. Cell 63: 405415.

Kitazono, A. and T. Matsumoto. 1998. 'Isogaba Maware': Quality control of genome DNA by checkpoints. BioEssays 20: 391-399.

Kumada, K., T. Nakamura, K. Nagao, H. Funabiki, T. Nakagawa, and M. Yanagida. 1998. Cutl is loaded onto the spindle by binding to Cut2 and promotes anaphase spindle movement upon Cut2 proteolysis. Curr. Biol. 8: 633-641.

Losada, A., M. Hirano, and T. Hirano. 1998. Identification of Xenopus SMC protein complexes required for sister chromatid cohesion. Genes \& Dev. 12: 1986-1997.

Michaelis, C., R. Ciosk, and K. Nasmyth. 1997. Cohesins: Chromosome proteins that prevent premature separation of sister chromatids. Cell 91: 35-45.

Molnar, M., J. Bahler, M. Sipiczki, and J. Kohli. 1995. The rec8 gene of Schizosaccharomyces pombe is involved in linear element formation, chromosome pairing, and sister-chromatid cohesion during meiosis. Genetics 141: 61-73.

Moore, D.P., A.W. Page, T.T. Tang, A.W. Kerrebrock, and T.L. Orr-Weaver. 1998. The cohesion protein MEI-S332 localizes to condensed meiotic and mitotic centromeres until sister chromatids separate. J. Cell Biol. 140: 1003-1012.

Moreno, S., J. Hayles, and P. Nurse. 1989. Regulation of p34 ${ }^{c d c 2}$ protein kinase during mitosis. Cell 58: 361-372.

Nabeshima, K., H. Kurooka, M. Takeuchi, K. Kinoshita, Y. Nakaseko, and M. Yanagida. 1995. p93 ${ }^{\text {disl }}$ required for sister chromatid separation is a novel microtubule and spindle pole body associating protein phosphorylated at the Cdc2 target sites. Genes \& Dev. 9: 1572-1585.

Nabeshima, K., S. Saitoh, and M. Yanagida. 1997. Use of green fluorescent protein for intracellular localization in living fission yeast cells. Methods Enzymol. 283: 459-471.

Nabeshima, K., Y. Nakagawa, A.F. Straight, A. Murray, Y. Chikashige, Y. Yamashita, Y. Hiraoka, and M. Yanagida. 1998. Dynamics in fission yeast Metaphase and Anaphase A: Constant spindle length period requiring Dis1 reveals distinct centromere movements. Mol. Biol. Cell 9: (in press).

Ohkura, H., N. Kinoshita, S. Miyatani, T. Toda, and M. Yanagida. 1989. The fission yeast dis $2^{+}$gene required for chromosome disjoining encodes one of two putative type 1 protein phosphatases. Cell 57: 997-1007.

Rothstein, R.J. 1983. One-step gene disruption in yeast. Methods Enzymol. 101: 202-211.

Rowles, A. and J.J. Blow. 1997. Chromatin proteins involved in the initiation of DNA replication. Curr. Opin. Genet. Dev. 7: 152-157.

Saitoh, S., K. Takahashi, and M. Yanagida. 1997. Mis6, a fission yeast inner centromere protein, acts during G1/S and forms specialized chromatin required for equal segregation. Cell 90: 131-143.

Saka, Y. and M. Yanagida. 1993. Fission yeast cut $^{+}$gene required for the onset of S-phase and the restraint of M-phase is identical to the radiation-damage repair gene $\mathrm{rad}^{+}$. Cell 74: 383-393.

Samejima, I. and M. Yanagida. 1994. Bypassing anaphase by fission yeast cut9 mutation: Requirement $\mathrm{cut}^{+} \mathrm{9}^{+}$gene to initiate anaphase. J. Cell Biol. 127: 1665-1670.

Seitz, L.C., K. Tang, W.J. Cummings, and M.E. Zolan. 1996. The rad9 gene of Coprinus cinereus encodes a proline-rich protein required for meiotic chromosome condensation and synapsis. Genetics 142: 1105-1117.

Straight, A.F., A.S. Belmont, C.C. Robinett, and A.W. Murray. 1996. GFP tagging of budding yeast chromosomes reveals that protein-protein interactions can mediate sister chroma- tid cohesion. Curr. Biol. 6: 1599-1608.

Takahashi, K., S. Murakami, Y. Chikashige, O. Niwa, H. Funabiki, and M. Yanagida. 1992. A low copy number central sequence with strict symmetry and unusual chromatin structure in the fission yeast centromere. Mol. Biol. Cell 3: 819-835.

Takahashi, K., H. Yamada, and M. Yanagida. 1994. Fission yeast minichromosome loss mutants mis cause lethal aneuploidy and replication abnormality. Mol. Biol. Cell 5: 1145-1158.

Uzawa, S. and M. Yanagida. 1992. Visualization of centromeric and nucleolar DNA in fission yeast by fluorescence in situ hybridization. J. Cell Sci. 101: 267-275.

Yamada, H., K. Kumada, and M. Yanagida. 1997. Distinct subunit functions and cell cycle regulated phosphorylation of 20S APC/cyclosome required for anaphase in fission yeast. J. Cell Sci. 110: 1793-1804. 


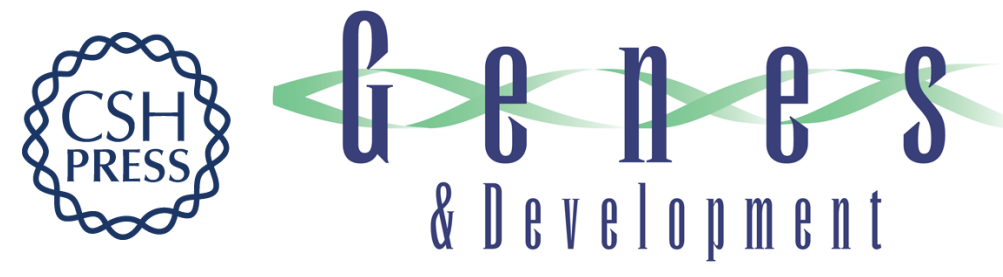

\section{Faithful anaphase is ensured by Mis4, a sister chromatid cohesion molecule required in $S$ phase and not destroyed in $G_{1}$ phase}

Kanji Furuya, Kohta Takahashi and Mitsuhiro Yanagida

Genes Dev. 1998, 12:

Access the most recent version at doi:10.1101/gad.12.21.3408

References This article cites 33 articles, 11 of which can be accessed free at: http://genesdev.cshlp.org/content/12/21/3408.full.html\#ref-list-1

License

Email Alerting

Receive free email alerts when new articles cite this article - sign up in the box at the top Service right corner of the article or click here.

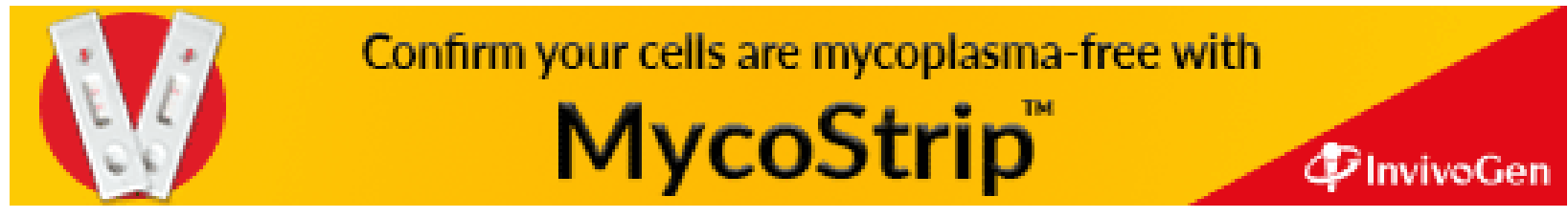

